\title{
Shift in sleep apnoea type in heart failure patients in the CANPAP trial
}

\author{
C.M. Ryan*, J.S. Floras*, A.G. Logan*, R.J. Kimoff", F. Series ${ }^{\ddagger}$, D. Morrison ${ }^{+}$, \\ K.A. Ferguson ${ }^{\S}$, I. Belenkie ${ }^{f}$, M. Pfeifer**, J. Fleetham ${ }^{\# \#}$, P.J. Hanly ${ }^{f}$, M. Smilovitch ${ }^{\#}$, \\ M. Arzt**** and T.D. Bradley* for the CANPAP Investigators ${ }^{* \pi}$
}

ABSTRACT: In patients with heart failure (HF), the predominant type of sleep apnoea can change over time in association with alterations in circulation time.

The aim of this study was to determine whether, in some patients with HF, a spontaneous shift from mainly central ( $>50 \%$ central events) to mainly obstructive ( $>50 \%$ obstructive events) sleep apnoea (CSA and OSA, respectively) over time coincides with improvement in left ventricular ejection fraction (LVEF). Therefore, sleep studies and LVEFs of HF patients with CSA from the control arm of the Canadian Continuous Positive Airway Pressure for Patients with Central Sleep Apnea and Heart Failure (CANPAP) trial were examined to determine whether some converted to mainly OSA and, if so, whether this was associated with an increase in LVEF.

Of 98 patients with follow-up sleep studies and LVEFs, 18 converted spontaneously to predominantly OSA. Compared with those in the nonconversion group, those in the conversion group had a significantly greater increase in the LVEF $(2.8 \%$ versus $-0.07 \%)$ and a significantly greater fall in the lung-to-ear circulation time (-7.6 s versus $0.6 \mathrm{~s})$.

In patients with HF, spontaneous conversion from predominantly CSA to OSA is associated with an improvement in left ventricular systolic function. Future studies will be necessary to further examine this relationship.

KEYWORDS: Central sleep apnoea, congestive heart failure, left ventricular function, obstructive sleep apnoea, sleep-disordered breathing

leep-disordered breathing, including obstructive and central sleep apnoea (OSA and CSA, respectively) is common in patients with heart failure (HF). The overall prevalence of sleep-disordered breathing in such patients has been reported to be between 45 and $72 \%$; that of CSA between 15 and $42 \%$; and that of OSA between 12 and 53\% [1-6].

Some patients with HF have a combination of both OSA and CSA on the same night. For example, in one study, TKACOVA et al. [7] described 12 HF patients who had approximately equal numbers of obstructive and central events during the same night. An intriguing observation was that in all 12 cases, the apnoea type shifted overnight from predominantly obstructive to predominantly central. Concurrently, there was an increase in circulation time and a reduction in carbon dioxide tension $\left(\mathrm{PCO}_{2}\right)$. These observations suggested that the shift from obstructive to central apnoea was related to an overnight deterioration in cardiac function. However, in that study, cardiac function was assessed only indirectly by measuring lung-to-ear circulation time (LECT). Nevertheless, these findings suggested that deterioration in cardiac function in HF patients with OSA might be accompanied by a shift in apnoea-type towards central predominance.

In another study, TKACOVA et al. [8] described a small group of patients with HF who converted from predominantly OSA on one night to predominantly CSA on another night at least 1 month later, and another group who converted in the opposite direction. In the first group, nocturnal $\mathrm{PCO}_{2}$ fell and the periodic breathing cycle duration increased, whereas, in the latter group, $\mathrm{PCO}_{2}$ increased and cycle duration decreased. In another study, improvement of cardiac function following cardiac transplantation in HF patients with CSA was accompanied by resolution of CSA in the majority, and conversion to predominantly OSA in a small minority [9]. However, because these latter subjects gained weight and underwent major changes in prescribed medications, it is not clear whether such conversion was related to improved cardiac function or to other factors. In
AFFILIATIONS

Depts of Medicine

*University of Toronto, Toronto, ON,

§University of Western Ontario,

London, ON,

\#McGill University, Montreal, QC,

-Laval University, Quebec City, QC

+Dalhousie University, Halifax, NS,

${ }^{f}$ University of Calgary, Calgary, $A B$, and

\#\#University of British Columbia,

Vancouver, BC, Canada.

**University of Regensburg,

Regensburg, Germany.

"A full list of the CANPAP

Investigators is provided in the

Acknowledgements.

\section{CORRESPONDENCE}

C.M. Ryan

Toronto General Hospital/

University Health Network,

$9 N-967$

200 Elizabeth Street

Toronto

ON

M5G 2C4

Canada

E-mail: clodagh.ryan@uhn.on.ca

Received:

April 302009

Accepted after revision:

Aug 062009 
view of these observations, we hypothesised that in $\mathrm{HF}$ patients with predominantly CSA, some would convert either spontaneously or as a result of intensified HF therapy to predominantly OSA, and such conversion would be accompanied by an improvement in cardiac function, as assessed both directly by measurement of the left ventricular ejection fraction (LVEF) and indirectly by measurement of LECT. To this end, we analysed prospective data from HF patients with CSA randomised to the control arm of the Canadian Continuous Positive Airway Pressure for Patients with Central Sleep Apnea and Heart Failure Trial (CANPAP) [10].

\section{METHODS}

\section{Study design}

CANPAP was a multicentre randomised, controlled open-label trial with blind analysis of end-points involving HF patients with CSA randomised either to optimal contemporary medical therapy [11] alone (control group) or with the addition of continuous positive airway pressure (CPAP) [10].

\section{Subjects}

Inclusion criteria were males and females aged 18-79 yrs who had New York Heart Association (NYHA) functional class II to IV HF due to ischaemic, hypertensive or idiopathic dilated cardiomyopathy and whose condition had been stabilised on optimal medical therapy for at least 1 month; an LVEF of $<40 \%$ on radionuclide angiography; and CSA, defined as $\geqslant 15$ apnoeas and hypopnoeas per hour of sleep (apnoea/hypopnoea index; $\mathrm{AHI}),>50 \%$ of which were central. Exclusion criteria were pregnancy, myocardial infarction, unstable angina or cardiac surgery within the previous 3 months, and OSA. Investigators were blind to the results of all follow-up assessments (including overnight sleep studies) until the termination of the trial. The research ethics boards of each of the 11 participating centres approved the protocol. Enrolment followed written informed consent.

Subjects from the control arm of CANPAP were included if they had both polysomnography and LVEF determined at either 3 months or 2 yrs after randomisation, as described below. Polysomnographic data and LVEF from the latest assessment for each patient were analysed and compared with baseline values.

\section{Baseline assessment}

Eligible patients underwent a baseline assessment of medications, body mass index (BMI), resting LVEF measured by radionuclide angiography and dyspnoea, assessed by the Chronic Heart Failure Questionnaire (CHFQ; which assesses patients' dyspnoea according to a seven-point scale, with lower scores indicating worse condition; changes $>0.75$ are considered clinically significant) $[12,13]$, followed by overnight polysomnography. Sleep stages and arousals, apnoeas and hypopnoeas, and mean and lowest arterial oxygen saturation $\left(\mathrm{Sa}_{1} \mathrm{O}_{2}\right)$ levels were assessed according to uniform criteria in all centres [13-16]. Respiratory efforts were measured by respiratory inductance plethysmography, and airflow by nasal pressure [14, 16, 17]. Central apnoea was defined as the absence of tidal volume for $\geqslant 10 \mathrm{~s}$ without thoracoabdominal motion, and central hypopnoeas as a reduction of $\geqslant 50 \%$ in tidal volume from baseline for $\geqslant 10 \mathrm{~s}$ with in-phase thoracoabdominal motion and without airflow limitation on nasal pressure. Apnoeas and hypopnoeas were classified as obstructive if there was out-of-phase motion of the rib cage and abdomen, or if airflow limitation on nasal pressure was present. The frequency of apnoeas and hypopnoeas per hour of sleep was quantified as the AHI.

In the subset of 22 patients from Toronto Site A (Toronto General Hospital/University Health Network, University of Toronto, Toronto, ON, Canada), in whom $\mathrm{Sa}_{1} \mathrm{O}_{2}$ was assessed by an ear, rather than a finger, oximeter, we analysed LECT during stage 2 nonrapid eye movement sleep as an estimate of lung-to-carotid chemoreceptor circulation time as previously described [18-20]. During episodes of recurrent central apnoeas, the first 10 consecutive periodic breathing cycles were analysed in each subject, which always occurred in the first half of the night. LECT was taken as the interval from the onset of the first breath terminating an apnoea to the nadir of the subsequent dip in $\mathrm{Sa}_{1} \mathrm{O}_{2}$. This technique has been validated against cardiac output as a measure of circulation time in patients with sleep apnoea, both with and without HF [20]. Body position was also recorded in this subset.

\section{Follow-up assessments}

Follow-up assessments of medications, LVEF, dyspnoea and polysomnographic variables were repeated at 3 and 24 months following randomisation for the entire cohort. For this analysis, the predominant type of sleep apnoea-hypopnoea was determined from the final polysomnogram available in conjunction with data from other tests performed at that visit. Those in whom $>50 \%$ of events remained central were classified as having CSA (i.e. nonconverters), while those in whom $\geqslant 50 \%$ of the events were obstructive were classified as having OSA (i.e. converters). In addition, in the subset of 22 patients from Toronto Site A, body position, BMI and LECT were also assessed at these times.

\section{Statistical analysis}

Baseline characteristics of patients in the two groups were compared by two-tailed unpaired t-tests for variables with normally distributed data, the Mann-Whitney rank sum test for variables with non-normally distributed data, and by Chisquared or Fisher's exact test for nominal variables as appropriate. To adjust for baseline differences, analysis of covariance was used to compare differences within and between groups in polysomnographic and cardiovascular variables, measured at baseline and at follow-up after enrolment. All data analyses were carried out with S-Plus 6.2 (Mathsoft Inc., Cambridge, MA, USA). All p-values are twosided.

\section{RESULTS}

\section{Characteristics of the patients}

Of the 258 patients enrolled in CANPAP, 130 were randomised to control and 128 to CPAP. Of the 130 patients randomised to the control, 32 were excluded because they died $(n=5)$, had a cardiac transplant $(n=1)$ or did not have a sleep study and/or an assessment of LVEF at 3 months or 2 yrs $(n=26)$. Of the 98 remaining patients, $18(18 \%)$ had converted to OSA on the last follow-up sleep study, while CSA persisted in the rest. Baseline characteristics of the 98 patients appear in table 1 . The mean $\mathrm{AHI}$ indicated a similar severe degree of CSA in both groups at baseline, with a similar proportion of both central and 
obstructive events. In both groups, HF was predominantly due to ischaemic heart disease. Patients in the conversion group were significantly younger, had a higher BMI, and had lower CHFQ dyspnoea scores and systolic blood pressure. More patients in the conversion group were on spironolactone than in the nonconversion group.

\section{Follow-up assessments}

Of the 98 patients included in the analyses, 56 had final followup polysomnography at 3 months and 42 at 2 yrs.

\section{Polysomnographic and respiratory data}

There were no significant changes in the mean and minimum $\mathrm{Sa}_{1} \mathrm{O}_{2}$ values, total sleep time, distribution of sleep stages or frequency of movement arousals between the baseline and follow-up assessments in either the nonconversion or conversion groups (table 2). As displayed in fig. 1, there was no

\begin{tabular}{|c|c|c|c|}
\hline & $\begin{array}{c}\text { Nonconversion } \\
\text { group }\end{array}$ & $\begin{array}{l}\text { Conversion } \\
\text { group }\end{array}$ & p-value \\
\hline Subjects & 80 & 18 & \\
\hline Age yrs & $64.3 \pm 1.0$ & $59.3 \pm 2.8$ & 0.05 \\
\hline Sex M:F & $76: 4$ & 18:0 & 1.00 \\
\hline BMI $\mathbf{k g} \cdot \mathbf{m}^{-2}$ & $28.5 \pm 0.6$ & $32.9 \pm 1.5$ & 0.002 \\
\hline$A H I n \cdot h^{-1}$ & $38.5 \pm 1.8$ & $39.1 \pm 4.2$ & 0.87 \\
\hline Cause of HF & & & 0.55 \\
\hline IDCM & $21(26)$ & $6(33)$ & \\
\hline Ischaemic & $56(70)$ & $11(61)$ & \\
\hline Hypertensive & $3(4)$ & $1(6)$ & \\
\hline LVEF & $24.3 \pm 0.9$ & $24.3 \pm 1.7$ & 0.99 \\
\hline NYHA class & & & 0.49 \\
\hline$\|$ & $59(74)$ & $13(72)$ & \\
\hline III & $20(25)$ & $4(22)$ & \\
\hline IV & $1(1)$ & $1(6)$ & \\
\hline \multicolumn{4}{|l|}{ Atrial fibrillation } \\
\hline Present & $13(16)$ & $3(17)$ & 1.00 \\
\hline \multicolumn{4}{|l|}{ Medications } \\
\hline Digoxin & $43(54)$ & $9(50)$ & 0.80 \\
\hline Loop diuretics & $72(90)$ & $16(89)$ & 1.00 \\
\hline Spironolactone & $27(34)$ & $12(67)$ & 0.02 \\
\hline ACE inhibitors & $63(79)$ & $17(94)$ & 0.18 \\
\hline$\beta$-blockers & $61(76)$ & $15(83)$ & 0.76 \\
\hline \multicolumn{4}{|l|}{ CHFQ score } \\
\hline Dyspnoea & $4.4 \pm 0.1$ & $3.5 \pm 0.2$ & 0.004 \\
\hline \multicolumn{4}{|c|}{ Blood pressure $\mathrm{mmHg}$} \\
\hline Systolic & $116 \pm 2$ & $103 \pm 3$ & 0.005 \\
\hline Diastolic & $71 \pm 1$ & $69 \pm 3$ & 0.51 \\
\hline
\end{tabular}

Data are presented as $n$, mean \pm SEM (for continuous values), or $n(\%)$, unless otherwise indicated. M: male; F: female; BMI: body mass index; $\mathrm{AHI}$ : apnoea/ hypopnoea index; HF: heart failure; IDCM: idiopathic dilated cardiomyopathy; LVEF: left ventricular ejection fraction; NYHA: New York Heart Association; ACE: angiotensin-converting enzyme; $\mathrm{CHFQ}$ score: Chronic Heart Failure Questionnaire score (lower scores indicate more dyspnoea). The nonconversion and the conversion groups were defined as having $\geqslant 50 \%$ central events and $>50 \%$ obstructive events, respectively. change in the total AHI, obstructive AHI or central AHI between baseline and follow-up in the nonconversion group (89\% central and 11\% obstructive events at both baseline and follow-up). However, there was a significant decrease in both the total AHI and central AHI, and, by definition, an increase in the obstructive AHI between the baseline and follow-up assessments in the conversion group (85\% central and $15 \%$ obstructive at baseline versus $27 \%$ central and $73 \%$ obstructive at follow-up). Between group differences were significant for the changes in the obstructive $(p<0.001)$ and central $(p<0.001)$ AHI but not the total AHI $(p=0.12)$. This shift involved both apnoea and hypopnoea types, although the shift in hypopnoea type was more pronounced. The events at baseline and followup were predominantly hypopnoeas in both the nonconversion (55\% to $55 \%$ ) and conversion group (65\% to $72 \%$; table 2 ).

In the subgroup of 22 subjects from Toronto Site A from baseline to last follow-up, there was no change in BMI in either the nonconversion $(\mathrm{n}=13,28.5 \pm 0.6$ to $28.4 \pm 1.0 ; \mathrm{p}=0.49)$ or conversion $(n=9,32.9 \pm 1.5$ versus $35.1 \pm 2.5 ; p=0.43)$ groups, $(\mathrm{p}=0.90$ for between group differences), and no significant change in the percentage of total sleep time spent in the supine position for either the nonconversion $(32.5 \pm 8.3 \%$ versus $27.1 \pm 8.2 \% ; \quad p=0.54) \quad$ or conversion $(55.1 \pm 10.2 \%$ versus $51.7 \pm 13.2 \%$; $p=0.86)$ groups $(p=0.912$ for between group differences). Whereas LECT did not change significantly from baseline to follow-up in the nonconversion group $(17.5 \pm 1.8$ versus $18.1 \pm 1.0 \mathrm{~s} ; \mathrm{p}=0.74)$, it decreased significantly by $7.6 \mathrm{~s}$ in the conversion group (from $20.6 \pm 3.4$ to $13.0 \pm 1.9 \mathrm{~s} ; \mathrm{p}=0.03$ ) (between group difference of $8.2 \mathrm{~s} ; \mathrm{p}=0.02$ ).

\section{Cardiovascular variables}

As shown in table 3, there were no significant between group differences in NYHA class from baseline to follow-up. However, there was a significantly greater increase in the mean LVEF from baseline to last follow-up in the conversion group than in the nonconversion group (between group difference of $3.5 \pm 0.6 \% ; p=0.01$ ). This difference in LVEF continued to be statistically significant after adjusting for BMI $(p=0.041)$. There was also a significantly greater improvement in the CHFQ dyspnoea score in the conversion than in the nonconversion group (between group difference of $0.9 \pm 0.1$; $\mathrm{p}=0.01)$ (table 2).

With respect to medications, in the nonconversion group, there were significant increases in the percentage of individuals prescribed $\beta$-blockers ( $76 \%$ versus $85 \%, p=0.04$ ) and spironolactone ( $34 \%$ versus $43 \%, \mathrm{p}=0.02)$ from baseline to follow-up, but no change in other medications. There were no significant changes in the number of individuals prescribed any medication from baseline to follow-up in the conversion group ( $\beta$ blockers $89 \%$ versus $83 \%$; spironolactone $67 \%$ versus $72 \%$ ) or in the dosage of medications prescribed over this time. Overall, there was a significantly higher proportion of individuals prescribed spironolactone in the conversion group than the nonconversion group $(\mathrm{p}=0.02)$.

\section{DISCUSSION}

This analysis of HF patients with CSA from the control arm of the CANPAP trial was performed to determine if the nature of sleep apnoea could change spontaneously over time in the setting of a prospective randomised trial and, if so, whether 
TABLE 2 Polysomnographic outcomes

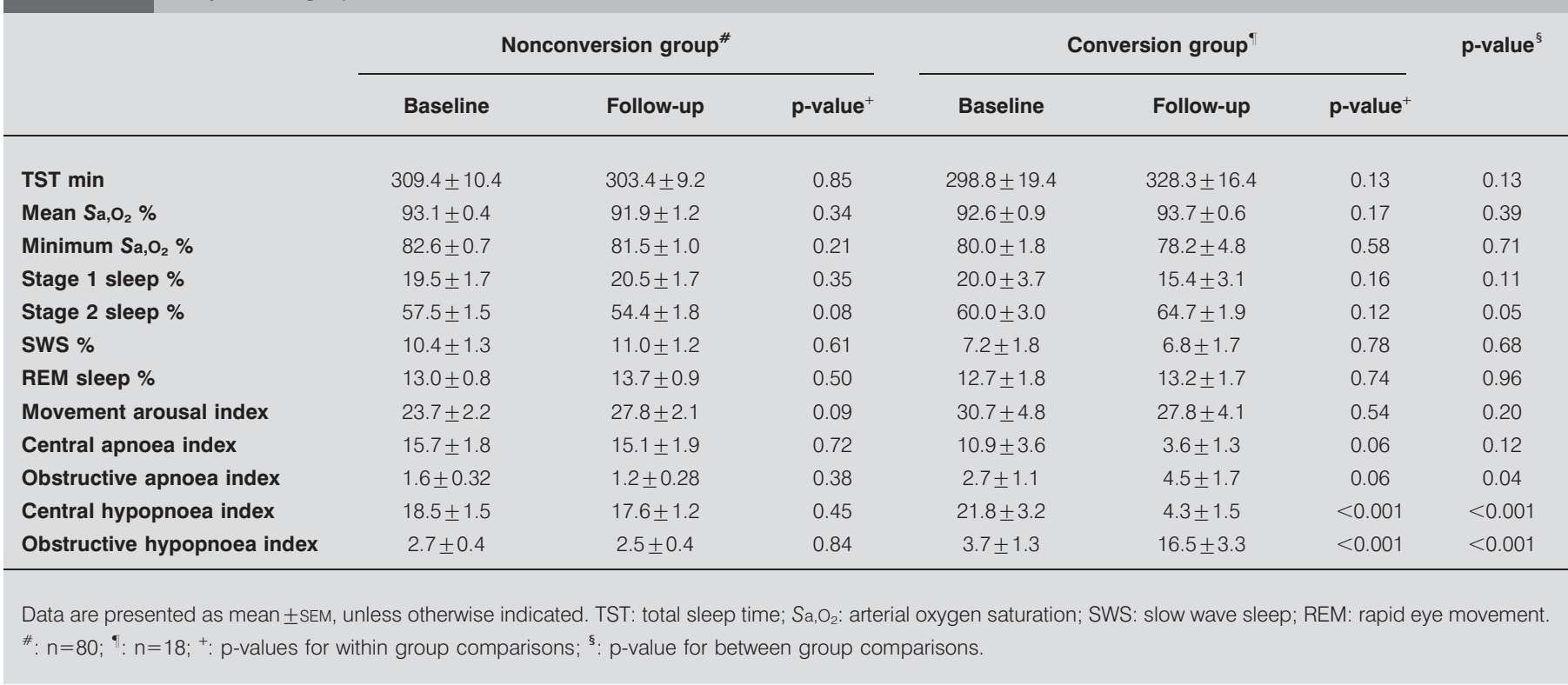

this was associated with changes in cardiac function or medications. We observed that in $18 \%$ of patients, there was a shift from predominantly CSA (85\% central events) to predominantly OSA (73\% obstructive events) over time and that this shift occurred in association with a significant improvement in cardiac function manifest by a 3.5\% increase in LVEF and an $8.2 \mathrm{~s}$ decrease in LECT compared with the

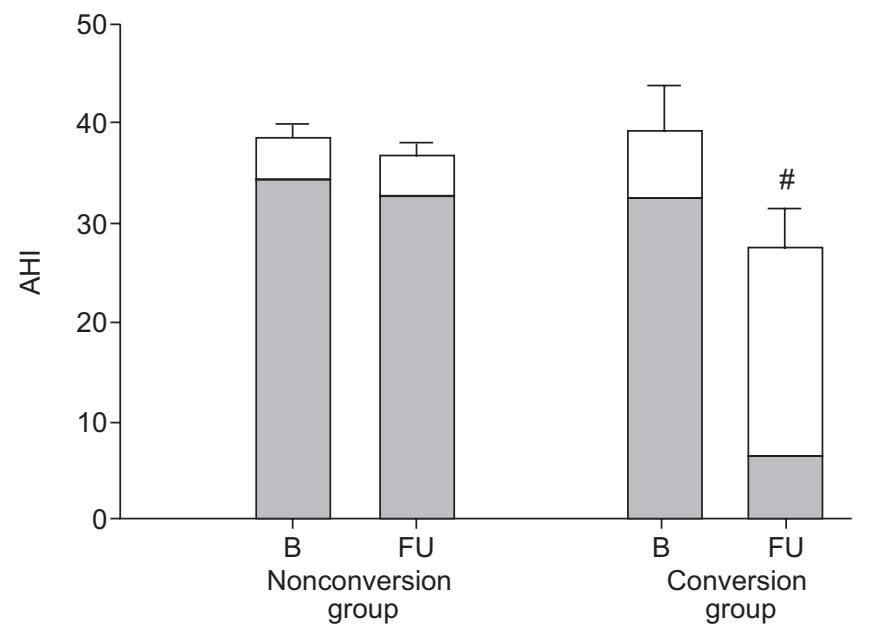

FIGURE 1. Total, central ( $\square$ ) and obstructive $(\square)$ apnoea/hypopnoea index $(\mathrm{AH})$ at baseline (B) and follow-up (FU) in the nonconversion and conversion groups. In the nonconversion group, there is no significant change in the total $(38.5 \pm 1.8$ versus $36.5 \pm 2.0 ; p=0.4)$, central $(34.3 \pm 1.8$ versus $32.7 \pm 2.0 ; p=0.49)$ or obstructive $(4.3 \pm 0.5$ versus $4.0 \pm 0.5 ; p=0.7) \mathrm{AHI}$ between baseline and followup. However, in the conversion group, there is a significant increase in the obstructive index ( $6.0 \pm 1.4$ versus $21.2 \pm 3.6 ; p=0.00)$ and a decrease in both the central $(33.1 \pm 3.8$ versus $7.7 \pm 1.2 ; p=0.00)$ and total $(39.1 \pm 4.2$ versus $28.9 \pm 4.4$; \#: $p=0.04) \mathrm{AHI}$ between baseline and follow-up. Between group differences are significant for the obstructive $(p<0.001)$ and central $(p<0.001) A H I$, but not for the total $(p=0.12) \mathrm{AHI}$. nonconversion group. However, conversion was not associated with any significant change in medications over this period.

The present observations are consistent with findings from the overnight and long-term studies by TKACOVA and CO-workers $[7,8]$. Data from those studies suggested that in patients with $\mathrm{HF}$, a shift from OSA to CSA is associated with worsening cardiac function, and vice versa. However, since those studies were retrospective in nature, and since investigators inferred cardiac function indirectly from LECT and periodic breathing cycle duration, their proposal that apnoea type may change in conjunction with a change in cardiac function remained unproven.

The present data, therefore, provide the first direct, prospective evidence that a shift from CSA to OSA in HF patients occurs in conjunction with an improvement in left ventricular systolic

\begin{tabular}{|c|c|c|c|}
\hline $\begin{array}{c}\text { TABLE } 3 \text { Ch } \\
\text { ba }\end{array}$ & $\begin{array}{l}\text { s in cardiova } \\
\text { to follow-up }\end{array}$ & variables fro & \\
\hline Variable & $\begin{array}{c}\text { Nonconversion } \\
\text { group }\end{array}$ & $\begin{array}{l}\text { Conversion } \\
\text { group }\end{array}$ & p-value \\
\hline Subjects $\mathrm{n}$ & 80 & 18 & \\
\hline$\Delta$ NYHA class & $0.0(-0.1-0.2)$ & $-0.1(-0.5-2.3)$ & 0.38 \\
\hline$\Delta$ LVEF \% & $-0.7(-1.9-0.6)$ & $2.8(-0.4-6.0)$ & 0.01 \\
\hline $\begin{array}{l}\triangle \mathrm{CHFQ} \text { dyspnoea } \\
\text { score }\end{array}$ & $0.1(0.5-1.2)$ & $0.9(0.2-1.6)$ & 0.01 \\
\hline
\end{tabular}

Data are presented as mean $(95 \% \mathrm{Cl})$, unless otherwise indicated. $\triangle \mathrm{NYHA}$ change in New York Heart Association; $\triangle \mathrm{LVEF}$ : change in left ventricular ejection fraction; $\triangle \mathrm{CHFQ}$ : change in Chronic Heart Failure Questionnaire score ( $>0.75$ represents important change of moderate magnitude). $p$-values are for between group comparisons (ANCOVA) 
function, assessed by LVEF. The concurrent reduction in LECT in a subgroup of these patients is consistent with the increase in LVEF and with an augmentation in cardiac output over time [20]. Furthermore, since our data are both prospective and are derived from the control arm of a randomised trial, they provide an estimate of how often this conversion might occur in the setting of optimal, contemporary HF treatment. We found that in those HF patients who converted from predominantly central to obstructive, the AHI, LVEF and NYHA class at baseline were similar to the nonconversion group. However, those patients with a shift from central to obstructive events were younger, more obese and more dyspnoeic as assessed by the CHFQ, and were more often prescribed spironolactone than their counterparts whose CSA persisted. The shift to OSA was accompanied by a reduction in dyspnoea that may be a clinical correlate of the increase in LVEF and decrease in LECT.

The reduction in LECT of $7.6 \mathrm{~s}$ may appear to be relatively greater than the absolute LVEF change of $2.8 \%$ in the conversion group. However, when the increase in LVEF is expressed as relative change, it is much greater at $12 \%$. In addition, most patients with systolic HF have functional mitral regurgitation that will cause overestimation of the LVEF, as it measures both forward and regurgitant flow. A reduction in mitral regurgitation causing an improvement in forward stroke volume may reduce LVEF [21]. Therefore, if mitral regurgitant fraction decreased, a small increase in LVEF would indicate a relatively large increase in nonregurgitant stroke volume and, therefore, be associated with a large decrease in LECT. Although, mitral regurgitation was not measured in CANPAP, a previous study demonstrated that treatment of CSA in HF patients with CPAP reduced mitral regurgitant fraction by $40 \%$. Therefore, the increase in LVEF due to CPAP observed in the present study probably underestimates the improvement in forward stroke volume [22]. Hence, the seeming discrepancy between the large decrease in the LECT versus the small increase in LVEF in the conversion group is probably attributable to a large relative increase in the LVEF and a concomitant reduction in the severity of mitral regurgitation.

It is not clear what underlying mechanisms were involved in the shift in predominant apnoea type in the conversion group. Intensification of medical therapy for HF is unlikely to be an explanation, since the conversion group had no significant increases in medications during the course of the trial. A change in weight is unlikely to be the explanation because there was no significant weight gain in the subgroup of the conversion group who had BMI assessed at baseline and follow-up. Although it is possible that left ventricular filling pressure decreased with a concomitant rise in $\mathrm{PCO}_{2}$ above the apnoea threshold $[18,23]$, resulting in conversion from CSA to OSA $[7,8]$, these variables were not measured during our study.

Another possibility is that CSA reverted to OSA that was present prior to the onset of HF. The greater weights of those who converted to OSA would be consistent with a greater predisposition to OSA. Previous studies suggest that in HF patients, OSA can convert to CSA in association with deterioration in indirectly assessed cardiac function $[7,8]$. It stands to reason that the opposite could occur, as observed in the present study. This is compatible with the observation that following cardiac transplantation in HF patients with CSA, CSA either resolves or converts to OSA [9]. A limitation of the study was that data on sleeping position and BMI on followups were not collected for the entire cohort as part of the protocol. However, in the subgroup of 22 subjects from Toronto Site A where these measurements were made, conversion from CSA to OSA was not associated with any significant change in sleeping position or BMI. These data suggest that conversion was not due to a greater proportion of time spent supine or an increase in weight. It has been shown that like OSA, CSA is more common in the supine than in the lateral positions $[24,25]$, so it is unlikely that a change in body position accounted for conversion from CSA to OSA.

In conclusion, we have demonstrated in a cohort of $98 \mathrm{HF}$ patients with CSA followed prospectively, that OSA emerges spontaneously over time in a substantial minority in association with an improvement in LVEF and a decrease in circulation time. These observations provide insights into the pathophysiology of sleep apnoea by suggesting that, at least in some patients with HF, OSA and CSA can be part of a spectrum of sleep-related breathing disorders whose predominant nature can change over time in association with alterations in cardiac function [7, 8].

\section{SUPPORT STATEMENT}

The CANPAP Trial was supported by an investigator-initiated, peerreviewed grant from the Canadian Institutes of Health Research (CIHR) through its University-Industry Program (UIP). In accordance with UIP policy, once the grant application was approved, one-third of the funding was provided by the CIHR and industry partners provided two-thirds. Because CPAP devices are generic, industry partnership was sought from all interested manufacturers. At the time of initial grant approval, four industry partners each agreed to provide $25 \%$ of the industry contribution, including donating their brand of CPAP device. However, prior to the onset of fund dispersal, one of the industry partners (Healthdyne) was acquired by another (Respironics). Therefore, Respironics took over the obligations of Healthdyne to the trial, thus providing $50 \%$ of the industry contribution.

J.S. Floras was supported by a Canada Research Chair in Integrative Cardiovascular Biology and a Career Investigator Award from the Heart and Stroke Foundation of Ontario. F. Series was supported by a National Scientist Award from the Fonds de la Recherche en Sante du Quebec. T.D. Bradley was supported by a Senior Scientist Award from the CIHR.

\section{CLINICAL TRIALS}

This study has been registered with www.controlled-trials.com (ISRCTN 25258560).

\section{STATEMENT OF INTEREST}

Statements of interest for C.M. Ryan, R.J. Kimoff, D. Morrison, K.A. Ferguson, M. Pfeifer, P.J. Hanly and M. Arzt and the study itself, can be found at www.erj.ersjournals.com/misc/statements.dtl

\section{ACKNOWLEDGEMENTS}

CANPAP Trial Administration. Executive Committee: T.D. Bradley (Chair), A.G. Logan and J.S. Floras. Advisory Committee: N. Anthonisen (Dept of Medicine, University of Manitoba, Winnipeg, MB, Canada) and E.R. Smith (Dept of Medicine, University of Calgary, Calgary, AB, Canada). Data and Safety Monitoring Committee: M. Bourassa (Chair; University of Montreal, Montreal, QC, Canada), 
D. Stewart (University of Toronto, Toronto, ON, Canada) and L. Magee (University of British Columbia, Vancouver, BC, Canada). End Point Adjudication Committee: J.S. Floras (Chair), I. Belenkie and J. Howlett (Dalhousie University, Halifax, NS, Canada).

CANPAP Sites and Investigators. I. Belenkie and W. Whitelaw (University of Calgary), M. Heule (University of Alberta, Edmonton, AB, Canada), D. Morrison and J. Howlett (Dalhousie University), K.A. Ferguson (University of Western Ontario, London, ON, Canada), R.J. Kimoff and M. Smilovitch (McGill University, Montreal), F. Series and M.H. Leblanc (Laval University, Quebec City, QC, Canada), T.D. Bradley, A.G. Logan, J.S. Floras, H. Ross, D. Delgado and R.S.T. Leung (University of Toronto, Site A: Toronto General Hospital/University Health Network) and P.J. Hanly (Site B: St Michael's Hospital), J. Fleetham, K. Gin and J. Wilson (University of British Columbia), S. Corne and E. Azevedo (University of Manitoba), and M. Pfeifer, S. Montalvan-Dobmayr and T. Schichtl (University of Regensburg, Regensburg, Germany).

CANPAP Trial Managers. F.S. Fitzgerald, N. Catherine and G. Hopkins (all at Toronto General Hospital of University Health Network, Toronto).

Data Management Centre: The Prosserman Centre for Health Research, Samuel Lunenfeld Research Institute of the Mount Sinai Hospital, University of Toronto (D. Ng, Data Manager).

Trial Statisticians. G. Tomlinson (University of Toronto) and R.K. Parkes (Samuel Lunenfeld Research Institute, Mount Sinai Hospital).

\section{REFERENCES}

1 Javaheri S, Parker TJ, Liming JD, et al. Sleep apnea in 81 ambulatory male patients with stable heart failure. Types and their prevalences, consequences, and presentations. Circulation 1998; 97: 2154-2159.

2 Sin DD, Fitzgerald F, Parker JD, et al. Risk factors for central and obstructive sleep apnea in 450 men and women with congestive heart failure. Am J Respir Crit Care Med 1999; 160: 1101-1106.

3 Solin P, Bergin P, Richardson M, et al. Influence of pulmonary capillary wedge pressure on central apnea in heart failure. Circulation 1999; 99: 1574-1579.

4 Findley LJ, Zwillich CW, Ancoli-Israel S, et al. Cheyne-Stokes breathing during sleep in patients with left ventricular heart failure. South Med J 1985; 78: 11-15.

5 Lofaso F, Verschueren P, Rande JL, et al. Prevalence of sleepdisordered breathing in patients on a heart transplant waiting list. Chest 1994; 106: 1689-1694.

6 Ferrier K, Campbell A, Yee B, et al. Sleep-disordered breathing occurs frequently in stable outpatients with congestive heart failure. Chest 2005; 128: 2116-2122.

7 Tkacova R, Niroumand M, Lorenzi-Filho G, et al. Overnight shift from obstructive to central apneas in patients with heart failure: role of $\mathrm{PCO}_{2}$ and circulatory delay. Circulation 2001; 103: 238-243.

8 Tkacova R, Wang H, Bradley TD. Night-to-night alterations in sleep apnea type in patients with heart failure. J Sleep Res 2006; 15: 321-328.
9 Mansfield DR, Solin P, Roebuck T, et al. The effect of successfu heart transplant treatment of heart failure on central sleep apnea. Chest 2003; 124: 1675-1681.

10 Bradley TD, Logan AG, Kimoff RJ, et al. Continuous positive airway pressure for central sleep apnea and heart failure. $N$ Engl J Med 2005; 353: 2025-2033.

11 Bradley TD, Logan AG, Floras JS. Rationale and design of the Canadian Continuous Positive Airway Pressure Trial for Congestive Heart Failure patients with Central Sleep Apnea CANPAP. Can J Cardiol 2001; 17: 677-684.

12 Guyatt GH, Nogradi S, Halcrow S, et al. Development and testing of a new measure of health status for clinical trials in heart failure. J Gen Intern Med 1989; 4: 101-107.

13 Naughton MT, Liu PP, Bernard DC, et al. Treatment of congestive heart failure and Cheyne-Stokes respiration during sleep by continuous positive airway pressure. Am J Respir Crit Care Med 1995; 151: 92-97.

14 Clark SA, Wilson CR, Satoh M, et al. Assessment of inspiratory flow limitation invasively and noninvasively during sleep. Am J Respir Crit Care Med 1998; 158: 713-722.

15 Rechtschaffen A, Kales A. A Manual of Standardized Terminology, Techniques and Scoring System for Sleep Stages of Human Subjects. Los Angeles, UCLA Brain Information Service/ Brain Research Institute, 1968.

16 Sleep-related breathing disorders in adults: recommendations for syndrome definition and measurement techniques in clinical research. The Report of an American Academy of Sleep Medicine Task Force. Sleep 1999; 22: 667-689.

17 Staats BA, Bonekat HW, Harris CD, et al. Chest wall motion in sleep apnea. Am Rev Respir Dis 1984; 130: 59-63.

18 Lorenzi-Filho G, Rankin F, Bies I, et al. Effects of inhaled carbon dioxide and oxygen on Cheyne-Stokes respiration in patients with heart failure. Am J Respir Crit Care Med 1999; 159: 1490-1498.

19 Ryan CM, Bradley TD. Periodicity of obstructive sleep apnea in patients with and without heart failure. Chest 2005; 127: 536-542.

20 Hall MJ, Xie A, Rutherford R, et al. Cycle length of periodic breathing in patients with and without heart failure. Am J Respir Crit Care Med 1996; 154: 376-381.

21 Stevenson LW, Bellil D, Grover-McKay M, et al. Effects of afterload reduction (diuretics and vasodilators) on left ventricular volume and mitral regurgitation in severe congestive heart failure secondary to ischemic or idiopathic dilated cardiomyopathy. Am J Cardiol 1987; 60: 654-658.

22 Tkacova R, Liu PP, Naughton MT, et al. Effect of continuous positive airway pressure on mitral regurgitant fraction and atrial natriuretic peptide in patients with heart failure. J Am Coll Cardiol 1997; 30: 739-745.

23 Lorenzi-Filho G, Azevedo ER, Parker JD, et al. Relationship of carbon dioxide tension in arterial blood to pulmonary wedge pressure in heart failure. Eur Respir J 2002; 19: 37-40.

24 Bradley TD, McNicholas WT, Rutherford R, et al. Clinical and physiologic heterogeneity of the central sleep apnea syndrome. Am Rev Respir Dis 1986; 134: 217-221.

25 Szollosi I, Roebuck T, Thompson B, et al. Lateral sleeping position reduces severity of central sleep apnea/Cheyne-Stokes respiration. Sleep 2006; 29: 1045-1051. 